ARTICLE

Received 8 Oct 2015 | Accepted 13 Dec 2015 | Published 21 Jan $2016 \quad$ DOl: 10.1038/ncomms10457

\title{
Novel base-pairing interactions at the tRNA wobble position crucial for accurate reading of the genetic code
}

\author{
Alexey Rozov ${ }^{1, \star}$, Natalia Demeshkina ${ }^{1, \star}$, Iskander Khusainov $^{1,2}$, Eric Westhof ${ }^{3}$, Marat Yusupov ${ }^{1}$ \\ \& Gulnara Yusupova ${ }^{1}$
}

Posttranscriptional modifications at the wobble position of transfer RNAs play a substantial role in deciphering the degenerate genetic code on the ribosome. The number and variety of modifications suggest different mechanisms of action during messenger RNA decoding, of which only a few were described so far. Here, on the basis of several $70 \mathrm{~S}$ ribosome complex $\mathrm{X}$-ray structures, we demonstrate how Escherichia coli tRNA ${ }^{\text {Lys }}$ Uu with hypermodified 5-methylaminomethyl-2-thiouridine $\left(\mathrm{mnm}^{5} \mathrm{~s}^{2} \mathrm{U}\right)$ at the wobble position discriminates between cognate codons $A A A$ and $A A G$, and near-cognate stop codon UAA or isoleucine codon AUA, with which it forms pyrimidine-pyrimidine mismatches. We show that $m n m^{5} s^{2} U$ forms an unusual pair with guanosine at the wobble position that expands general knowledge on the degeneracy of the genetic code and specifies a powerful role of tRNA modifications in translation. Our models consolidate the translational fidelity mechanism proposed previously where the steric complementarity and shape acceptance dominate the decoding mechanism.

\footnotetext{
${ }^{1}$ Department of Integrated Structural Biology, Institute of Genetics and Molecular and Cellular Biology, INSERM, U964; CNRS/University of Strasbourg, UMR7104, 1 rue Laurent Fries, BP 10142, Illkirch 67404, France. ${ }^{2}$ Institute of Fundamental Medicine and Biology, Kazan Federal University, Karl Marx 18, Kazan 420012, Russia. ${ }^{3}$ Architecture and Reactivity of RNA, Institute of Molecular and Cellular Biology of the CNRS, University of Strasbourg, UPR9002, 15 rue Rene Descartes, Strasbourg 67084, France. ${ }^{\star}$ These authors contributed equally to the work. Correspondence and requests for materials should be addressed to G.Y. (email: gula@igbmc.fr).
} 
M ore than a hundred of posttranscriptional RNA modifications identified today ${ }^{1}$ were shown to play diverse and indispensable roles in gene regulation in all domains of life ${ }^{2}$. Modifications of RNA are carried out by complex cellular pathways, which involve countless protein enzymes and catalytic RNA-protein complexes, which primarily target tRNAs and, to a lesser extent, ribosomal RNA and mRNAs ${ }^{1}$. The observed trends suggest that many modification motifs and their sequence locations are conserved throughout Bacteria, Archaea and Eukarya; however, some kingdom-specific differences are documented as well.

Among all modifications found, those of tRNA are the most abundant and studied classes of modification $s^{1,3-5}$. Ninety-three tRNA modifications described today represent an astonishing library of chemically diverse structures ${ }^{5}$ each of which influences in a unique way three-dimensional integrity of the tRNA and specifies its physicochemical properties ${ }^{6,7}$. The most elaborate tRNA modifications are located in the anticodon loop, which comprises the anticodon triplet necessary for pairing with mRNA codons. The anticodon loops of almost all tRNAs contain several modified nucleotides. Among these, the most important are nucleotides in the positions 34 and 37. The nucleotide in position 34 (so-called 'wobble' position) pairs with the third mRNA codon base in the aminoacyl-tRNA binding site (A-site) during decoding $^{4,8}$. The nucleotide in the position 37 is adjacent to the $3^{\prime}$-side of the anticodon.

From the moment of their discoveries, modifications in tRNA anticodon loops were demonstrated to be crucial for proper mRNA decoding and fine-tuning of the process ${ }^{9}$. In particular, modifications of tRNA position 34 were implied to increase tRNA capacities to decode multiple mRNA codons differing by the third nucleoside (synonymous codons), hence explaining how the degenerate genetic code is translated ${ }^{4,10,11}$. It was shown also that anticodon modifications enhance recognition by corresponding aminoacyl-tRNA synthetases ${ }^{12,13}$ and serve as a preventing measure of frame shifting during translocation ${ }^{14,15}$. Very recently, the tRNA modifications were also credited a role in connecting translation, metabolism and stress response in bacteria and eukaryotes ${ }^{2,16}$. For example, in humans the deregulation of RNA modification pathways was shown to be linked to the type two diabetes and several mitochondrial diseases ${ }^{16}$.

Except for methionine and tryptophan, all amino acids are encoded by more than one codon, because the genetic code is redundant ${ }^{17,18}$ with 61 codons encoding 20 amino acids. In contrast to eukaryotes, where synonymous codons AAA and AAG are read by three isoacceptor lysine tRNA ${ }^{\text {Lys }}$, half of all bacteria have only one isoacceptor tRNA ${ }_{\text {UUU }}^{\text {Lys }}$ that decodes these two codons into amphipathic amino acid lysine ${ }^{9,19,20}$. To discriminate against pyrimidine-ending codons $\mathrm{AAC}$ and $\mathrm{AAU}$ encoding asparagine, the E. coli tRNA ${ }^{\mathrm{Lys}}$ UUU contains one of the most complex modifications 5-methylaminomethyl-2-thiouridine $\left(\mathrm{S}, \mathrm{mnm}^{5} \mathrm{~s}^{2} \mathrm{U}\right)$ at the wobble anticodon position 34 (Fig. 1a). The $\mathrm{mnm}^{5} \mathrm{~s}^{2}$ modification is a result of a sophisticated pathway that includes many enzymes responsible for thiolation and attachment of a methylaminomethyl group ${ }^{21}$. Another prominent feature of E. coli $\mathrm{tRNA}^{\mathrm{Lys}}$ sUU is N6-threonylcarbamoyladenosine $\left(\mathrm{t}^{6} \mathrm{~A}\right)$ at the 37th position of its anticodon loop (Fig. 1a). The $t^{6} \mathrm{~A}$ modification is one of the most ubiquitous and conserved, and is known to be critical for recognition of codons starting with adenosine. This is one of the rare modifications universally conserved throughout different kingdoms of life ${ }^{1}$. In addition to S34 and $\mathrm{t}^{6} \mathrm{~A} 37$, E. coli $\mathrm{RRNA}^{\mathrm{Lys}}$ suU anticodon loop bears the third modification, pseudouridine at position 39 (Fig. 1a).

Nuclear magnetic resonance studies of unmodified, partially and fully modified anticodon stem loops (ASLs) of E.coli
tRNA ${ }^{\text {Lys }}$ SuU demonstrated that $m n m^{5} s^{2} U$ and $t^{6} A$ modifications remodel an otherwise dynamic loop to canonical open U-turn structure to perfectly adapt in the ribosomal decoding centre ${ }^{6,22}$. First, X-ray studies of the partial decoding system ${ }^{23}$, where crystals of the isolated small ribosomal $30 \mathrm{~S}$ subunit were soaked with synthetic ASL of E.coli tRNA ${ }_{\text {Lys }}^{\text {LUU }}$ and hexaribonucleotides as mRNA analogues, shed some light on possible roles of modifications in decoding 24,25 . It was suggested that $t^{6} \mathrm{~A} 37$ enhances the codon-anticodon stability via cross-strand stacking interaction with the first codon nucleotide, whereas partially modified $\mathrm{mnm}^{5} \mathrm{U} 34$ lacking 2-thio group was implicated in an alternate $\mathrm{mnm} \mathrm{m}^{5} \mathrm{U} 34 \bullet \mathrm{G}$ base-pairing interactions via a bifurcated hydrogen bond ${ }^{25}$. A similar model of the bacterial 30 S subunit with ASL of human $\mathrm{tRNA}_{3}{ }^{\text {Lys }}{ }_{\text {UUU, }}$, which has identical anticodon loop sequence with $E$. coli tRNA ${ }^{\mathrm{Lys}}$ suU but carries $\mathrm{ms}^{2} \mathrm{t}^{6} \mathrm{~A} 37$ (2-methylthio-N6-threonylcarbamoyladenosine) and $\mathrm{mcm}^{5} \mathrm{~s}^{2} \mathrm{U} 34$ (5-methoxycarbonylmethyl-2-thiouridine) modifications, revealed Watson-Crick-like geometry of $\mathrm{mcm}^{5} \mathrm{~s}^{2} \mathrm{U} 34 \bullet \mathrm{G}$ base-pairing interactions at the wobble codon-anticodon position ${ }^{26}$. Both of these works and numerous other genetic, biophysical and biochemical findings indicated that each group in a modified nucleotide improves thermodynamic properties of tRNA and serves to augment specific codon-anticodon interactions during decoding.

Recent crystallographic studies of the $30 \mathrm{~S}$ ribosomal subunit or complete $70 \mathrm{~S}$ ribosome complexes with different ASLs ${ }^{23-27}$ or tRNAs ${ }^{28-33}$ and mRNAs describe three major classes of preferred geometry at the wobble position of a codon-anticodon minihelix. The first class consists of canonical Watson-Crick purinepyrimidine $\mathrm{A} \bullet \mathrm{U}\left(\right.$ or $\mathrm{U} \bullet \mathrm{A}$ ) and $\mathrm{C} \bullet \mathrm{G}\left(\right.$ or $\mathrm{G} \bullet \mathrm{C}$ ) pairs ${ }^{29,30,33}$, and also includes Watson-Crick-like pairs. It was suggested that the latter closely resemble canonical geometry via stabilization of an enol tautomer by the wobble modifications (for example, above described $\left.\mathrm{mcm}^{5} \mathrm{~s}^{2} \mathrm{U} 34 \bullet \mathrm{G}\right)^{26,27}$. The second class consists of standard wobble pair G34•U originally predicted by $\mathrm{Crick}^{8}$. In this pair, the pyrimidine is displaced towards the major groove of the codon-anticodon minihelix; however, the distance between the ribose $\mathrm{Cl}^{\prime}$ atoms remains very close to $10.5 \AA$, the average value for a standard Watson-Crick pair ${ }^{28,29}$. The third class

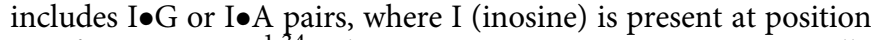
34 of some tRNAs ${ }^{1,34}$. These purine-purine pairs are unusually wide with a $\mathrm{Cl}^{\prime}-\mathrm{Cl}^{\prime}$ distance of $12.3 \AA$ (refs 24,32 ).

In the current study, we describe six X-ray structures of physiologically relevant complexes of the complete $70 \mathrm{~S}$ ribosome primed with long mRNAs that contain full-length native E. coli $\mathrm{tRNA}^{\mathrm{fMet}}$ in the peptidyl-tRNA-binding site (P-site) and tRNA ${ }^{\text {Lys }}$ SuU bound to cognate or near-cognate codons in the A-site. We identified an unprecedented base-pairing interaction at the wobble position of the codon-anticodon duplex in the decoding centre that broadens the present family of 'wobble geometries'. This base pair, which involves a hypermodified S34 of E. coli tRNA ${ }^{\text {Lys }}$ suU and codon guanosine, represents a 'wobble' $(\mathrm{G} 34 \bullet \mathrm{U})$ with the $\mathrm{U}$ moved towards the minor instead of the major groove that is much less isosteric to its flipped form than usual wobble $\mathrm{G} \bullet \mathrm{U}$ pair.

The ribosome structures we are describing in this work deepen the understanding of the tRNA discrimination mechanism on the ribosome. We demonstrate how tRNA ${ }^{\text {Lys }}$ suU discriminates between cognate codons AAA and AAG, and the near-cognate stop codon UAA (ochre codon) or the isoleucine codon AUA, with which it forms pyrimidine-pyrimidine $\mathrm{U} \bullet \mathrm{U}$ mismatches. Together with our earlier structures of the $70 \mathrm{~S}$ ribosome with various mismatches in the codon-anticodon duplex ${ }^{29,30}$, the present models expand our library of various states of the $70 \mathrm{~S}$ decoding centre. The present evidence further strengthens our proposition that the steric complementarity is predominant over 

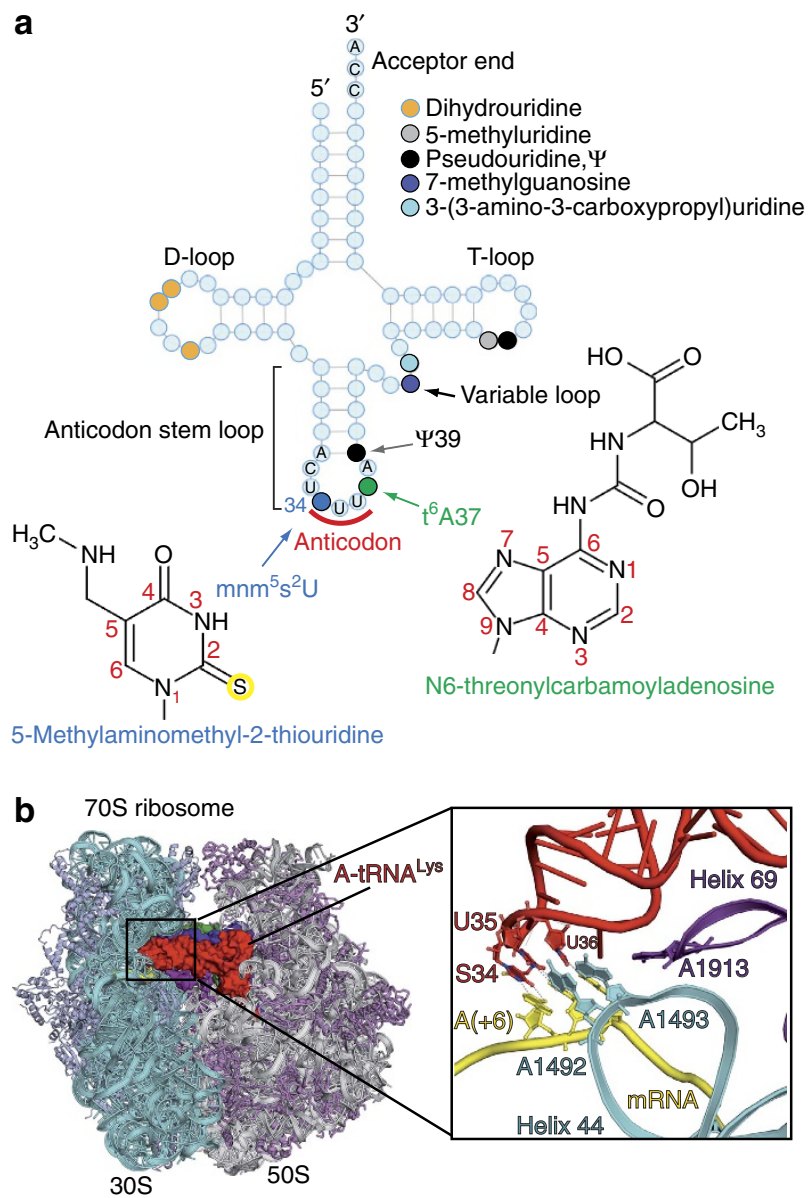

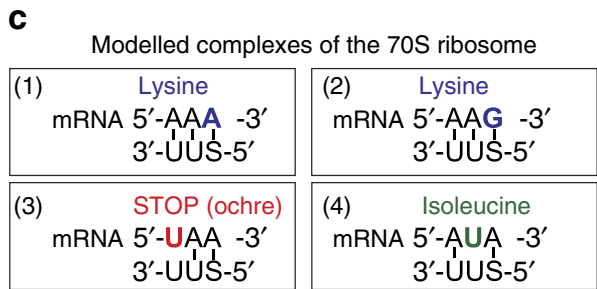

S - $m n m^{5} \mathrm{~s}^{2} U$

(3) and (4) without or with paromomycin

Figure 1 | Structure and modifications of lysine tRNA ${ }^{\text {Lys }}$ suu from $E$. coli and complexes of the 70 S ribosome with tRNA ${ }^{\text {Lys }}$ suu in the A-site.

(a) Secondary structure of E. coli tRNA ${ }^{\text {Lys }}$ suu. Major domains and modifications of tRNA are indicated; chemical formulas of hypermodified nucleobases at positions 34 and 37 are given together with corresponding abbreviations. (b) Side view of the 70S ribosome complex with three tRNAs bound at the A- (red), P- (blue) and exit (green) binding sites. Helix 69 of the large subunit is in magenta. The frame designates the decoding centre with the bound anticodon-stem loop of tRNA ${ }^{\text {Lys }}$ suu and the close-up view on the codon-anticodon duplex and major nucleotides of the decoding centre (G530 from 16S rRNA is not shown) including A1913 from 23S rRNA. (c) Schemes of codon-anticodon duplexes in the decoding centre of the 70 S ribosome complexes modelled in the study. The complexes are numbered in accordance with description in the main text.

the number of hydrogen bonds ${ }^{35}$ between the decoding centre and the codon-anticodon duplex, and hence plays the crucial discriminatory role during decoding.

\section{Results}

The modifications of lysine tRNA in the 70S decoding centre. In this work as in our previous studies ${ }^{28-30}$, we employed the full Thermus thermophilus $70 \mathrm{~S}$ ribosome co-crystallized with long synthetic mRNAs and natural tRNAs. These complexes model cognate or near-cognate states of the decoding centre at the proofreading step of the tRNA selection process (Fig. 1b). We determined six X-ray structures of the $70 \mathrm{~S}$ ribosome programmed by 30-nucleotide-long mRNAs with AUG codon and $E$. coli tRNA $^{\text {fMet }}$ in the P-site and the A-site occupied by tRNA ${ }^{\text {Lys }}$ suU bound to its cognate codon AAA or AAG, or near-cognate stop codon UAA and isoleucine codon AUA (Fig. 1c and Table 1).

The electron density maps of the two cognate complexes (Fig. 1c, complexes 1 and 2) possess sufficient level of detail to discern structural features of the tRNA anticodon loops that can be attributed to the influence of modifications $\mathrm{mnm}^{5} \mathrm{~s}^{2} \mathrm{U}$ and $\mathrm{t}^{6} \mathrm{~A}$ (Fig. 2). The amino group of the modified nucleotide S34 forms a hydrogen bond with $2^{\prime}-\mathrm{OH}$ of nucleotide U33 (Fig. 2a), thus altering the U-turn structure and stabilizing it, as was shown before for isolated ASLs ${ }^{6}$. The thio group of the same nucleotide is known to stabilize $3^{\prime}$-endo conformation of the ribose favourable for base-pairing interactions ${ }^{36}$, influencing the codon-anticodon helix stability. We observed a feature, characteristic of 2-thiouridine ${ }^{37}$ as well, namely the S2(S34)N1(U35) 'stacking' interaction with the subsequent nucleotide U35 (Fig. 2a). This interaction affects the relative positioning of the S34 and U35 nucleotides, and hence the shape and stability of the codon-anticodon duplex.

An influence of the large 50S ribosomal subunit on the tRNA constraints during decoding remained underestimated for a long time, because the first models of decoding were based on the structures of the isolated small ribosomal subunit ${ }^{23,35}$. On the $70 \mathrm{~S}$ ribosome, the conserved helix 69 of the 50S subunit, which is pivotal for many functions of the ribosome, directly contacts the sugar moiety of the tRNA nucleotide at position 37 (refs 31,38 and Fig. 2b). Most probably, this contact is important for proper positioning and conformational stabilization of the anticodon loop. In addition, $\mathrm{t}^{6} \mathrm{~A} 37$ forms cross-strand stacking with the first nucleotide of the mRNA codon in the A-site (Fig. 2c). Similar stacking interactions were described in the early models of the $30 \mathrm{~S}$ subunit whose crystals were soaked with the tRNA ${ }^{\mathrm{Lys}}$ UUU ASL carrying $\mathrm{t}^{6} \mathrm{~A} 37$ and $\mathrm{mnm}{ }^{5} \mathrm{U} 34$ modifications ${ }^{25}$. However, the 
Table 1 | Data collection and refinement statistics.

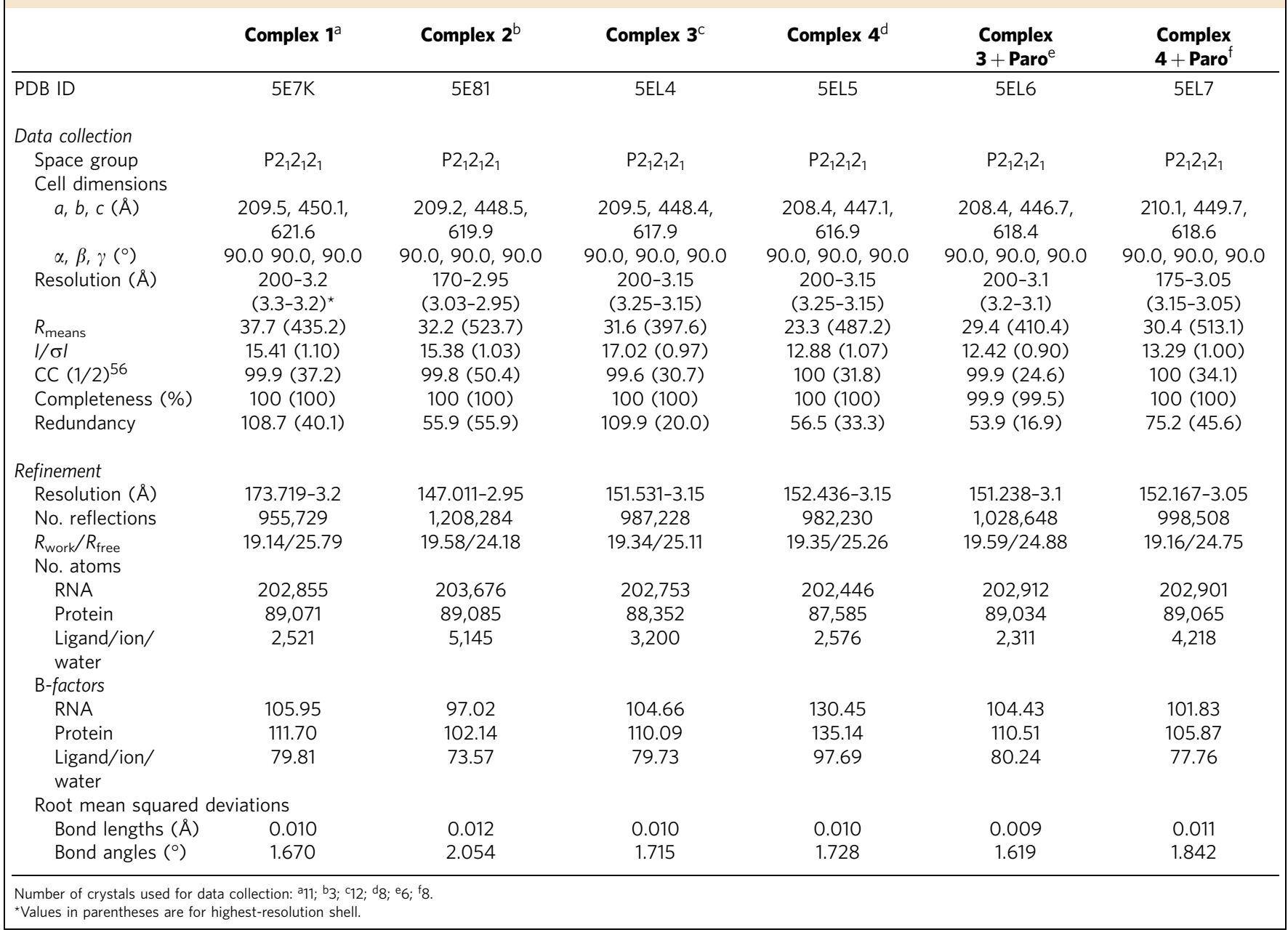

comparison of our present structure with this model revealed a considerable shift of $1 \AA$ in the position of the $t^{6} \mathrm{~A} 37$ nucleotide pointing to a specific role of the large $50 \mathrm{~S}$ subunit in restraining the anticodon loop of the A-site bound tRNA (Supplementary Fig. 1). The position of $t^{6} A 37$ over the A $\bullet U$ pair itself (Fig. 2c), as observed in our structures, corresponds well with the main function of this modification in stabilizing the weak $\mathrm{A} \bullet \mathrm{U}$ basepairing interactions and preventing mRNA slippage during translocation as well.

Hypermodified uridine forms a unique base pair. The capacity of $E$. coli tRNA $^{\text {Lys }}$ suU to read both codons AAA and AAG ending with purines implies that the hypermodified uridine S34 at the first anticodon position is involved in a dual mode of base-pairing interactions with adenosine and guanosine. In general, in bacteria the AAA codon is used approximately three times more often than the AAG codon ${ }^{39}$. However, it was estimated that when the next codon after the one encoding lysine starts with cytidine the AAG codon becomes preferred ${ }^{40}$.

Our first structure with E. coli tRNA ${ }^{\mathrm{Lys}}$ suU bound to its cognate AAA codon showed that hypermodified uridine S34 formed a distorted Watson-Crick base pair with the opposing adenosine with the standard $\mathrm{Cl}^{\prime}-\mathrm{Cl}^{\prime}$ distance of $10.6 \AA$ (Fig. 3a). The slight positional deviation of the uracil ring from its standard position in the Watson-Crick $\mathrm{A} \bullet \mathrm{U}$ pair, caused by the interactions of the modifications with neighbouring nucleotides
U33 and U35 (Fig. 2a), resulted in weakening of interaction between the codon adenosine and S34. On the other hand, the very same interactions tend to strengthen the codon-anticodon duplex as a whole by adjustment of the shape of tRNA U-turn ${ }^{6}$ (Fig. 2a).

The model of tRNA ${ }^{\text {Lys }}$ suu bound to its second cognate codon AAG demonstrated an unprecedented and striking base-pairing geometry (Fig. 3b). The new $\mathrm{S} 34 \bullet \mathrm{G}(+6)$ pair is characterized by the larger $\mathrm{Cl}^{\prime}-\mathrm{Cl}^{\prime}$ distance of $11.5 \AA$ that exceeds a corresponding distance in a standard Watson-Crick pair by $1 \AA$. Yet, interatomic distances between Watson-Crick edges within S34•G $(+6)$ imply the existence of two hydrogen bonds between carbonyl oxygen and N3 atom of modified uracil, and N3 atom and amino group of guanosine, respectively (Fig. 3b).

One of the hypotheses that could explain observed pairing interactions suggested that under physiological conditions a significant fraction of $\mathrm{mnm}^{5} \mathrm{~s}^{2} \mathrm{U}$ is present in a zwitterionic form (Fig. $4 \mathrm{a})^{41}$. This is due to the increased acidity of the N3 proton by the inductive effect of the protonated methylaminomethyl group, as was predicted based on the theoretical estimate of the $\mathrm{p} K_{\mathrm{a}}$ (ref. 41). In its neutral form, the modified uracil forms two hydrogen bonds same as in the case for a standard Watson-Crick U•A pair and as we observed for the $\mathrm{mnm}^{5} \mathrm{~s}^{2} \mathrm{U} 34 \bullet \mathrm{A}(+6)$ pair (Fig. 4b). In its deprotonated form, the N3 atom of the uracil becomes a proton acceptor and can form a hydrogen bond with the amino group of guanosine, while the uracil carboxyl group is engaged in another hydrogen bond 
a

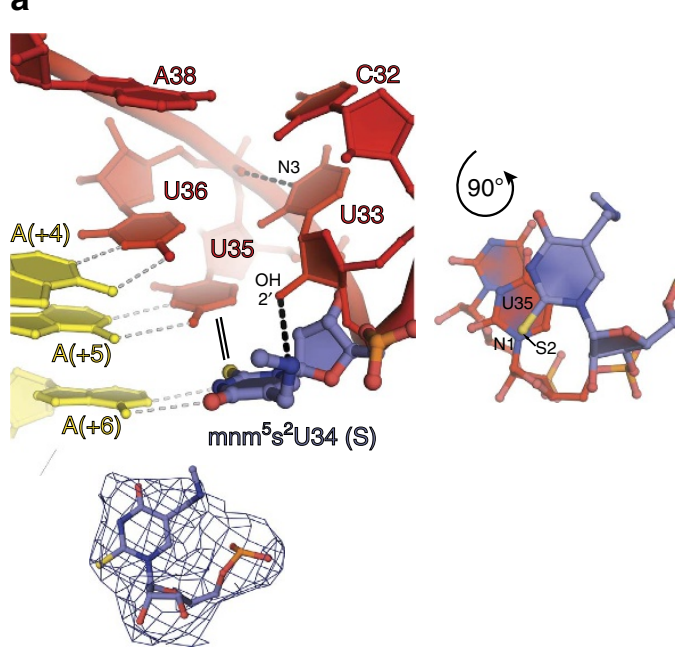

C b

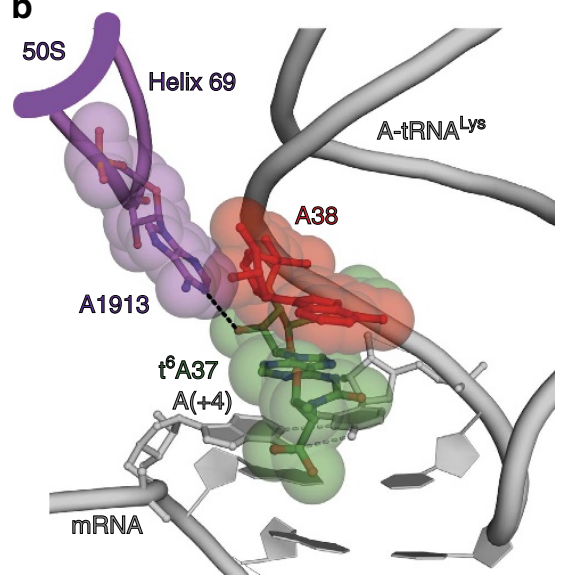

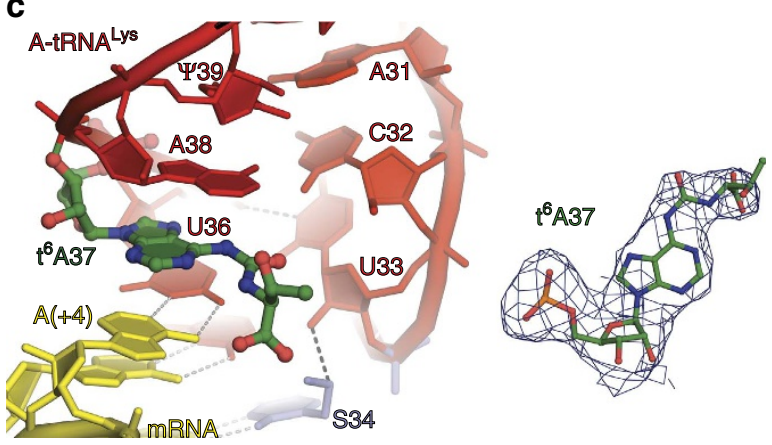

Figure 2 | Details of $\mathbf{E}$. coli tRNA ${ }^{\text {Lys }}$ suu anticodon loop interactions. (a) The S34 interactions: stabilization of the U-turn structure via anchoring of the $\mathrm{mnm}^{5}$ group and stacking of the thio group with the N1 atom of U35 shown in an alternative top-view orientation on the right. Nucleotide 37 is omitted for clarity of representation. (b) The invariant A1913 in Helix 69 of $23 \mathrm{~S}$ rRNA of the large ribosomal subunit (thick magenta line) constrains position of the $\mathrm{t}^{6} \mathrm{~A} 37$ ribose by conserved hydrogen bond interactions; van der Waals surfaces show that $\mathrm{A} 1913$ also defines conformation of the anticodon loop from the $3^{\prime}$-side of $t^{6} \mathrm{~A} 37$ at position 38. (c) Cross-strand stacking of $t^{6} \mathrm{~A} 37$ with the first nucleotide of the A-site bound codon (position ( +4 )). In a and $\mathbf{c}$, $2 F_{\mathrm{o}}-F_{\mathrm{c}}$ electron density maps corresponding to $\mathrm{S} 34$ and $\mathrm{t}^{6} \mathrm{~A} 37$ are contoured at $1.0 \sigma$.

with the guanosine N3 atom (Fig. 4c). Although less probable, the observed unusual pattern of hydrogen bonds between the modified uridine and guanosine can also be rationalized by existence of either rare tautomeric states (Fig. 4d,e) or an alternative zwitterionic state (Fig. $4 \mathrm{f}$ ) of the modified uridine.

It is worth to underline here that in contrast to the first two codon-anticodon positions, which are tightly restricted by the decoding centre ${ }^{29,30}$, the 'wobble' pair is not very firmly stabilized. The codon nucleotide is held in place only by indirect interactions with G530, C518 and S12 through a $\mathrm{Mg}^{2+}$ ion (Fig. 3a,b) and the O4' of the first anticodon nucleotide forms weak off-centre lone pair- $\pi$ interaction with the nucleobase of C1054 in 16S rRNA (Supplementary Fig. 2) ${ }^{42}$. However, the fact that observed $\mathrm{S} 34 \bullet \mathrm{G}(+6)$ pair is distorted from the standard 'wobble' geometry suggests that the aforementioned indirect restraints and interactions of the modification groups provide some restraints to control geometry of the third base pair.

Pyrimidine-pyrimidine mismatch in the 705 decoding centre. The translation of genes into proteins is an error-prone process with the average frequencies of mistranslation $10^{-3}-10^{-5}$ (ref. 43). We have recently published first structural rationales for the phenomenon of translational infidelity ${ }^{30}$. We demonstrated that because the $\mathrm{G} \bullet \mathrm{U}$ mismatches can mimic the form of a canonical Watson-Crick pair via tautomerization or ionization, these type of mismatches become accepted in the decoding centre, which restricts geometries of allowed pairs to canonical interactions. At the same time, our models with the $\mathrm{A} \bullet \mathrm{A}$ and C•A mismatches at the first two positions of the codonanticodon duplex suggested that these pairs would be efficiently discriminated against because of (i) steric clashes within a mispair or of a mispair with the tight decoding centre itself, or (ii) because of absence of stable pairing interactions between pairing nucleotides ${ }^{29,30}$.

In the current study, we asked an ensuing question of what is the structural basis for discrimination against the pyrimidinepyrimidine $\mathrm{U} \bullet \mathrm{U}$ pair, which represents a low-probability mistake during translation ${ }^{44-46}$. Thus, we determined structures of complexes 3 and 4 (Fig. 1c) where the SUU anticodon of tRNA $^{\text {Lys }}$ SUU formed a U $\bullet U$ mismatch either with the first or the second codon position. As was anticipated, the nucleotides, critical for decoding A1493 and A1492/G530 of 16S rRNA ${ }^{47}$, stabilized the $\mathrm{U} \bullet \mathrm{U}$ mismatch at the first and the second codonanticodon positions via A-minor groove interactions (Fig. 5a). These results substantiated our expanded mechanism of decoding that provides structural basis for discrimination in favour of correct tRNAs and against incorrect tRNAs, and describes identical rearrangements of the decoding centre on binding of cognate or near-cognate tRNA ${ }^{29,30}$. In both complexes, the interatomic distances between the Watson-Crick edges of opposing uracils exceeded $3.4 \AA$, implying weak electrostatic 
a

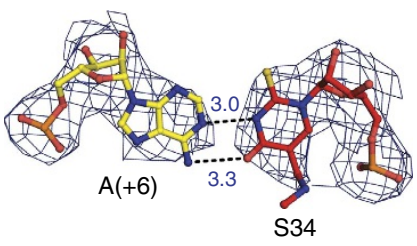

b
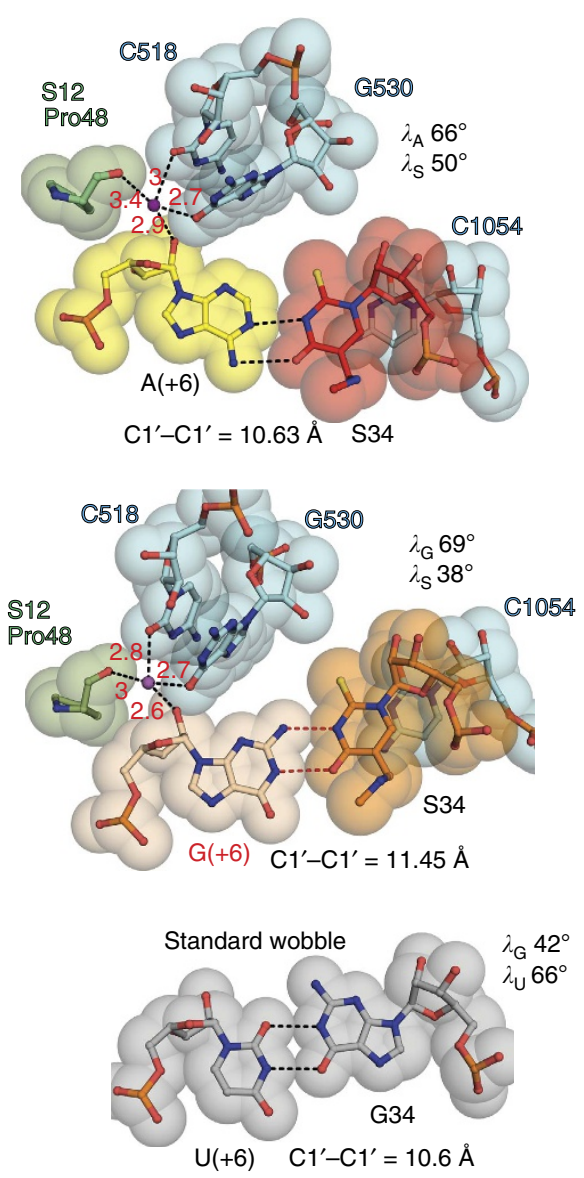

C

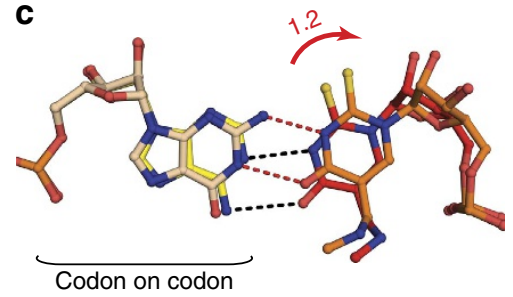

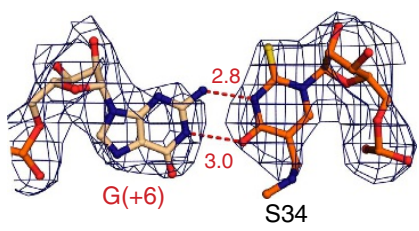

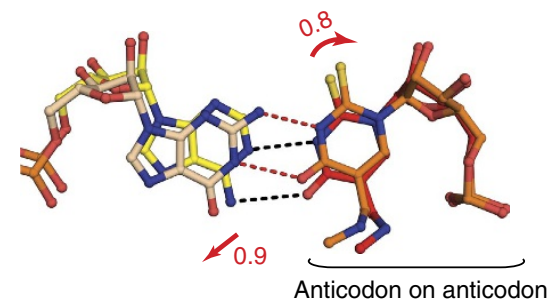

Figure 3 | Base pairs at the wobble position of the codon-anticodon duplex formed by the SUU anticodon of E. coli tRNA ${ }^{\text {Lys }}$ suU on cognate codons AAA or AAG. (a) The $S 34 \bullet A(+6)$ pair. Left, hydrogen bonds are indicated $(\AA)$; right, van der Waals surfaces with the corresponding $C 1^{\prime}-C 1^{\prime}$ distance and glycosidic angles $(\lambda)$. (b) The $\mathrm{S} 34 \bullet \mathrm{G}(+6)$ pair; indicated parameters as in $\mathbf{a}$; possible hydrogen bonds are marked by red dashes. In $\mathbf{a}$ and $\mathbf{b}$, the sugar moiety of codon nucleosides is coordinated by magnesium ion (shown in magenta) together with small subunit elements (Pro 48 of S12 and C518 with G530 in 16S rRNA); coordination distances show that the position of the wobble nucleotide in the codon can slightly adjust depending on the type of pairing interactions. The anticodon nucleotide is weakly restricted by $\mathrm{C} 1054$ in $16 \mathrm{~S}$ rRNA via lone pair-aromatic interactions. The lower panel displays the standard wobble G34•U( +6$)$ pair with specified parameters as in $\mathbf{a}$ and $\mathbf{b}$; see PDB code: $3 \mathrm{H} 8 \mathrm{l}$. (c) Superposition of $\mathrm{S} 34 \bullet A(+6)$ and $\mathrm{S} 34 \bullet \mathrm{G}(+6)$ pairs by the mRNA codons (left) or by the tRNA ${ }^{\text {Lys }}$ suu anticodon (right) shows relative extent of the guanosine and modified uracil displacement in the $\mathrm{S} 34 \bullet \mathrm{G}(+6)$ pair; the displacement is estimated by angstroms and marked by red arrows. In $\mathbf{a}$ and $\mathbf{b} 2 F_{\mathrm{o}}-F_{\mathrm{c}}$ electron density maps are contoured at $1.5 \sigma$.

interactions (Fig. 5b,c). A hypothetical U•U pair possible through a shift in the keto-enol equilibrium would require a typical distance of 3.0-3.1 Å between uracils for hydrogen bonds to occur (Fig. 5d). However, positions of uracils in both models make this scenario unlikely (Fig. 5b,c). To put it simply, the restraints on the sugar-phosphate backbones of the codon-anticodon helix imposed by the decoding centre are strong enough to prevent the uracils to come close enough to interact strongly via hydrogen bonds (Supplementary Fig. 3).

In complex 3 with the stop codon UAA and the first $\mathrm{U} \bullet \mathrm{U}$ mismatch, the codon-anticodon duplex was additionally weakened at the $3^{\prime}$-end where the $\mathrm{S} 34 \bullet \mathrm{A}(+6)$ pair was slightly deformed (Fig. 5e). As a result, only three strong hydrogen bonds were formed between codon and the anticodon compared with the cognate version with six hydrogen bonds (Fig. 5f). These

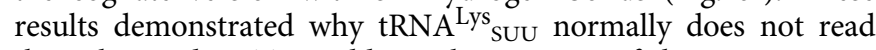
the ochre codon. Two additional structures of the near-cognate complexes 3 and 4 (Fig. 1c) solved in the presence of antibiotic paromomycin, which stimulates miscoding, supported our previous conclusion of the antibiotic mechanism of action ${ }^{48}$. In both cases, paromomycin stabilized A1492 and A1493 in the 'out' from the interior of helix 44 positions, hence stimulating A-minor groove interactions with the first two nucleotides of the A-codon. In addition, binding of the antibiotic led to a positional shift of the A1493 phosphate, resulting in partial alleviation of the restrictive decoding centre from the side of the mRNA codon. Finally, in the ribosome structures with paromomycin we observed a displacement of helix 69 of the large subunit 

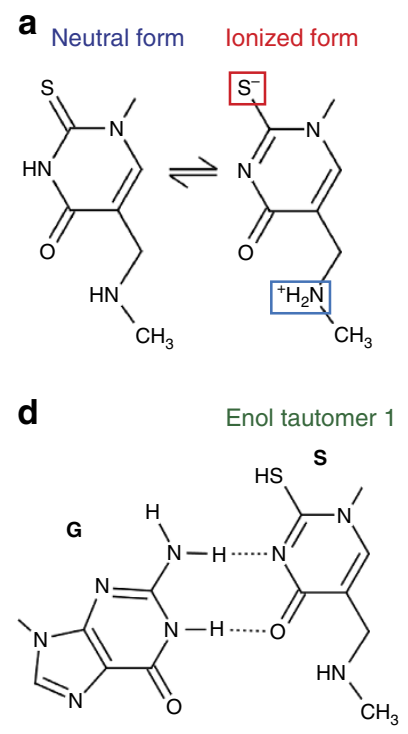

b<smiles></smiles>

e<smiles></smiles>

C

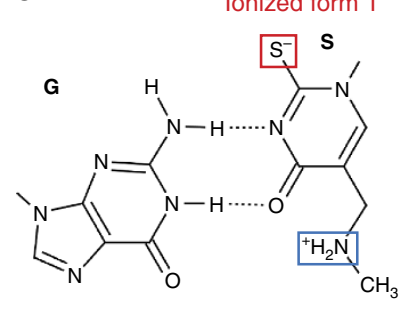

f

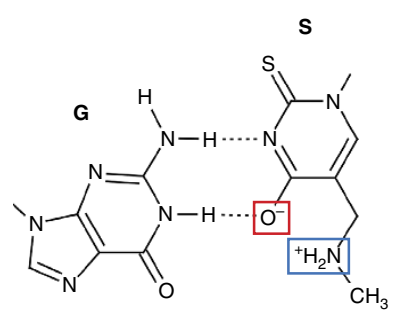

Figure 4 | The chemical basis of observed base-pairing interactions of S34 with the third codon adenosine or guanosine. (a) Equilibrium between two forms of $\mathrm{mnm}^{5} \mathrm{~s}^{2} \mathrm{U}^{41}$. (b) Interactions with adenosine of the neutral form of $\mathrm{mnm}^{5} \mathrm{~s}^{2} \mathrm{U}$. (c) Theoretically predicted base pair with guanosine of zwitterionic form of $\mathrm{mnm}^{5} \mathrm{~s}^{2} \mathrm{U}$ carrying negative charge on sulfur atom. (d,e) Two alternative pairing interactions with guanosine and enol tautomeric forms of $\mathrm{mnm}^{5} \mathrm{~s}^{2} \mathrm{U}$. (f) Base-pairing interactions with guanosine of a possible zwitterionic form of $\mathrm{mnm}^{5} \mathrm{~s}^{2} \mathrm{U}$.

towards the $\mathrm{D}$-stem of $\mathrm{tRNA}^{\mathrm{Lys}}$ suU that, most probably, enhanced stabilization of a near-cognate substrate on the ribosome.

It is important to mention that uracils in a mismatch at the first codon-anticodon position were closer to each other than at the second position (Fig. 5b,c). In the light of kinetic description of the tRNA selection process on the ribosome, it can be interpreted as the second codon-anticodon position being more controlled than the first one ${ }^{43}$. Accordingly, kinetic evaluations of codon readings by $\mathrm{tRNA}^{\mathrm{Lys}}$ UUU in bacteria assigned the highest accuracy values for the second position in the codon-anticodon duplex ${ }^{45}$. Thus, it is important to indicate here that despite of the fact that we could crystallize described near-cognate states of the ribosome at the proofreading step, in solution these complexes would be prone for dissociation because of a prominent lack of pairing codon-anticodon interactions.

\section{Discussion}

Fifty years ago, Francis Crick suggested some rules for translation of the genetic code on the ribosome and postulated the wobble hypothesis that gave first explanations to degeneracy of the code $^{8,18}$. It was predicted that the first two positions of the codon would pair with the anticodon using the standard base pairs, while in the base pairing of the third codon base 'there is a certain amount of play, or wobble, such that more than one position of pairing is possible'. First examples of foretold wobble nonstandard pairs included $\mathrm{G} \bullet \mathrm{U}, \mathrm{U} \bullet \mathrm{G}, \mathrm{I} \bullet \mathrm{A}, \mathrm{I} \bullet \mathrm{C}$ and $\mathrm{I} \bullet \mathrm{U}$ pairs, where the nucleoside on the left designates position 34 in tRNA ${ }^{8}$. Since those times, a titanic work on deciphering the genetic code resulted into a simple textbook chart where most of the codons are two- or fourfold degenerate, meaning that one amino acid can be coded by two or four codons differing by the third base. Further identification of tRNA modifications and especially those at the first anticodon 'wobble' position 34 elaborated more on the phenomenon of degeneracy. The 'modified wobble hypothesis' suggested that specific tRNA base modifications evolved to discriminate particular codons-expanding and facilitating an ability of tRNA to read more than one codon in some cases and preventing misreading in other cases ${ }^{4}$.
In the present study, we describe a new type of a base pair at the third wobble position of a codon-anticodon duplex in the $70 \mathrm{~S}$ ribosome decoding centre. Our structures demonstrate that the reversed 'wobble' pair $\mathrm{S} 34 \bullet \mathrm{G}(+6)$ adopts its own geometry, different from the standard $\mathrm{G} 34 \bullet \mathrm{U}(+6)$ pair ${ }^{8}$ at the third codon-anticodon position (Fig. 3b). This is possibly due to both certain restraints put on the third base pair by the decoding centre of the $70 \mathrm{~S}$ ribosome and modifications on the nucleotide S34 of tRNA ${ }_{\text {Sys }}^{\text {LyU }}$, shaping the codon-anticodon helix and the ASL of the tRNA (see Results). In spite of the fact that $\mathrm{S} 34 \bullet \mathrm{G}(+6)$ is not isosteric to the standard wobble pair, there is a certain similarity of the overall shape between these two pairs.

Both pairs are characterized by displacement of the anticodon nucleotide-S34 in the $S 34 \bullet G(+6)$ pair and $G$ in the $\mathrm{G} 34 \bullet \mathrm{U}(+6)$ pair-towards the minor groove of the codonanticodon minihelix. This is achievable because of the apical location of the nucleotide in the U-turn structure of the tRNA anticodon loop ${ }^{49}$. On the codon side of the pairs there is another tendency of shifting; however, in this case it is towards the major groove of the minihelix. Results reported in this study particularly show this displacement (Fig. $3 \mathrm{c}$ right). Hence, a term 'wobble' can be also applied to the capacity of the third codon nucleotide to adjust its position because of difference in strength and type of restraints imposed by the decoding centre on the third base pair as compared with the first and second base pairs (Fig. 3a,b).

Uridine at the wobble positions of various tRNAs is almost always modified in bacteria and eukaryotes ${ }^{1,5}$. In many cases, tRNAs with the modified uridines read two codons ending with purine $A$ or $G$ and, in some rare cases, modifications help to recognize all four nucleotides $A, G, C$ and $U$ at the third codon position ${ }^{50}$. Previously, it was shown that preferential form of the third wobble pair with a fully modified uridine approached a standard Watson-Crick-like geometry if this uridine was paired with guanosine $e^{26,27}$ (Supplementary Fig. 4). Thus, in the partial and heterologous model of the isolated bacterial $30 \mathrm{~S}$ ribosomal subunit, whose crystals were soaked with an ASL of human tRNA $_{3}$ Lys ${ }_{\text {UUU, }}$, the modified $\mathrm{mcm}^{5} \mathrm{~s}^{2} \mathrm{U} 34$ formed C•G-like $\mathrm{mcm}^{5} \mathrm{~s}^{2} \mathrm{U} 34 \bullet \mathrm{G}$ pair $^{26}$. In this pair, the uridine base was shifted towards the major groove when compared with geometry of the 

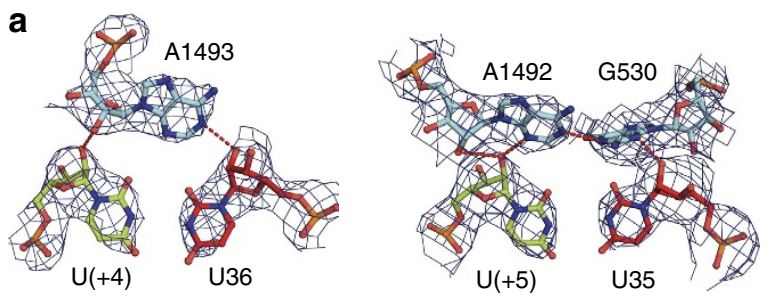

b
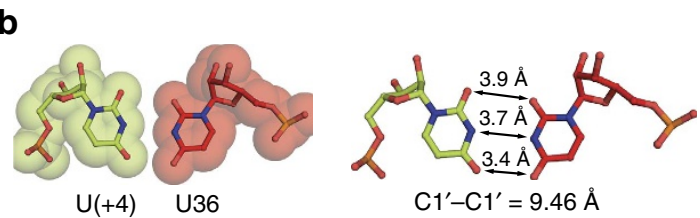

Main hydrogen bond categories
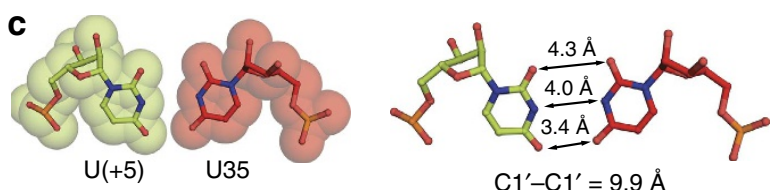

$$
C 1^{\prime}-C 1^{\prime}=9.9 \AA
$$

d

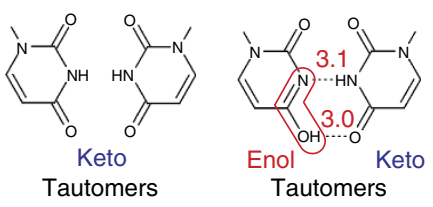

e

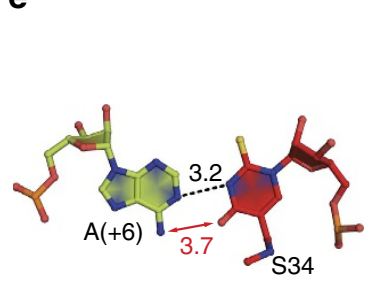

f Near-cognate duplex:

3 hydrogen bonds

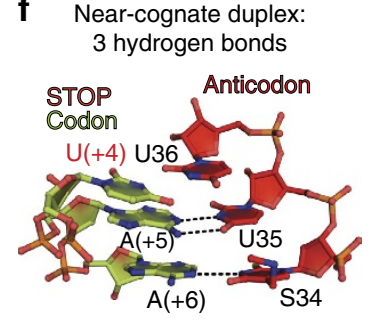

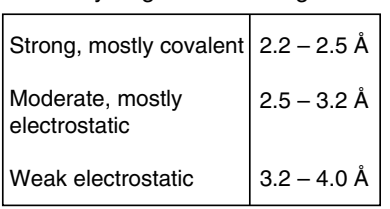

\begin{tabular}{l|l} 
Weak electrostatic & $3.2-4.0 \AA$ \\
\hline
\end{tabular}

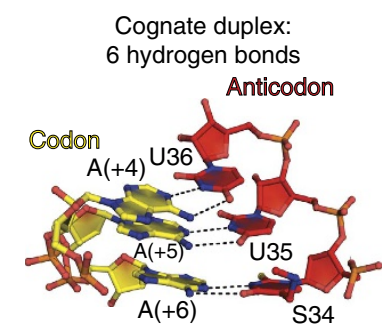

Figure 5 | Discrimination basis against near-cognate duplexes with pyrimidine-pyrimidine U•U mismatches in the decoding centre of the $70 S$

ribosome. (a) Critical A1492 and A1492/G530 of 16S rRNA stabilize the sugar phosphate backbones of the U•U mismatch at the first (left) and second (right) positions of the codon-anticodon duplex by A-minor groove interactions as is the case for any canonical Watson-Crick pair $29,30.2 F_{\mathrm{o}}-F_{\mathrm{c}}$ electron density maps are contoured at $1.5 \sigma .(\mathbf{b}, \mathbf{c})$ Geometries of the $\mathrm{U} \bullet U$ mismatch at the first $(\mathbf{b})$ and second (c) positions of the codon anticodon duplex; van der Waals surfaces (left) together with interatomic distances (right) are presented. In $\mathbf{b}$, the table describes standard categories of hydrogen bonds.

(d) Formation of a strong $\cup \bullet U$ pair would necessitate a shift in the keto-enol equilibrium from abundant keto form (left panel) to a rare enol form (red frame) and the $3-\AA \AA$ distance between Watson-Crick surfaces of opposing uridines (right panel). (e) Conformation of the wobble S34•A( +6$)$ pair in the complex 3 (see Fig. 1c). The absence of two hydrogen bonds expected for the $\mathrm{mnm}^{5} \mathrm{~s}^{2} \mathrm{U} \bullet \mathrm{A}$ pair can reflect (i) a deformation of the codon-anticodon minihelix induced by the mismatch and (ii) the specific influence of the tRNA modification at position 34 known to counteract misreading of the genetic code. (f) The near-cognate duplex composed of the tRNA ${ }^{\text {Lys }}$ suu anticodon and the ochre stop codon is significantly weakened compared with the cognate version on the AAA codon with the full set of canonical Watson-Crick interactions. The described weakening of the near-cognate duplex would imply dissociation of tRNA ${ }^{\text {Lys }}$ SUU from the ribosome or, in other terms, rejection.

$m n m^{5} s^{2} \mathrm{U} \bullet \mathrm{G}(+6)$ pair in our model (Supplementary Fig. $4 \mathrm{~b}$ left). In another study, uridine-5-oxyacetic acid at the position 34 of tRNA ${ }^{\mathrm{Val}}$ displayed a similar Watson-Crick-like pair ${ }^{27}$ with guanosine greatly displaced towards the minor groove of a codon-anticodon helix when compared with our structure (Supplementary Fig. 4c left). On the basis of these $30 \mathrm{~S}$ models, it was reasoned that modifications stabilized enol tautomers of uracil to keep the Watson-Crick-like geometry. It should also be mentioned here that although these structures gave important insights into how tRNA modifications can influence base-pairing interactions in the decoding centre, they described a partial system of decoding where roles of a full-length tRNA and the large ribosomal subunit could not be taken into consideration. At the same time, proper understanding of most often subtle fine-tuning effects of tRNA modifications on the codon-anticodon pairing geometries would require advanced experimental system consisting of full-length ligands and complete ribosome.

The novel pairing interaction at the third position of the codon-anticodon duplex described in this work deepens our understanding of principles embedded into translation of the genetic code on the ribosome. In agreement with the 'modified wobble hypothesis', our data show that the shape of the 'wobble' base pair is jointly defined by the ribosome environment and the tRNA modifications. In the observed case, such synergy gave rise to the novel base-pairing pattern, never observed before in the tRNA-mRNA duplexes. From the observation we can derive both a wider spatial tolerance of the wobble base-pair environment than expected before and, on the other hand, a certain degree of 
strictness imposed on the base pair, forcing it into the conformation unusual for a relaxed duplex.

Together with our preceding models describing $\mathrm{G} \bullet \mathrm{U}, \mathrm{U} \bullet \mathrm{G}$, $\mathrm{A} \bullet \mathrm{C}$ and $\mathrm{A} \bullet \mathrm{A}$ mismatches in codon-anticodon duplexes bound in the $70 \mathrm{~S}$ decoding centre, the present models with pyrimidinepyrimidine $\mathrm{U} \bullet \mathrm{U}$ mismatches consolidate the translation fidelity mechanism put forward by us earlier ${ }^{29,30}$. In this mechanism, the ribosome responds identically on binding of cognate or nearcognate tRNA by enveloping the codon-anticodon duplex in a rigid universal mould of the 'closed' decoding centre, which favours the Watson-Crick geometry of codon-anticodon base pairs. It is crucial to emphasize that when near-cognate tRNA with a mismatch to the mRNA codon binds to the decoding centre (during initial selection and proofreading steps), the number of hydrogen bonds between the minor groove of the near-cognate codon-anticodon helix and the 'closed' decoding centre elements is the same as would be the case during binding of the cognate tRNA. Therefore, the ribosome is incapable to distinguish between these states by the minor groove geometry of codon-anticodon base pairs. However, in contrast to cognate tRNAs that will stably pair to the mRNA codon by canonical Watson-Crick interactions, near-cognate tRNAs with a mismatch to the codon will be more likely to dissociate from the ribosome because of the strict restraints imposed by the tertiary structure of tRNA and elements of the 'closed' decoding centre on the codonanticodon helix. This pressure constitutes the discriminatory force by preventing the following conformational changes: (a) widening of the codon-anticodon helix needed to accommodate bulky non-canonical pairs (for example, A•A pair); (b) narrowing of the codon-anticodon helix necessary for proper pairing interaction in pyrimidine-pyrimidine pairs (for example, U•U pair); or (c) shift of nucleobases towards minor or major groove, characteristic of, for example, wobble $\mathrm{G} \bullet \mathrm{U}$ pair. The third base pair of the codon-anticodon duplex contributes in a different manner, compared with the first two positions. Its role in decoding is linked to the base pair nature, the indirect restraints imposed by the decoding centre and the presence of the modification groups that influence conformations of the tRNA ASL and the codon-anticodon helix. An additional discriminatory role at the proofreading step can be performed by the tails of some ribosomal proteins that selectively stabilize cognate tRNA substrates ${ }^{51}$. The rare translational mistakes caused by the incorporation of near-cognate tRNAs are reasoned mostly by the ability of some tRNAs to form Watson-Crick-like base pairs via ionization or tautomerism ${ }^{29}$, or in some cases a mismatch randomly escapes discrimination by preserving geometry close to the Watson-Crick pair ${ }^{30}$. The present decoding mechanism further establishes that discrimination between tRNAs is primarily founded on spatial fit $^{29,30,48}$ rather than on the number of hydrogen bonds between the 'closed' decoding centre and the codon-anticodon duplex ${ }^{35}$.

\section{Methods \\ Materials. Uncharged, native individual tRNA ${ }^{\mathrm{Lys}}{ }_{\mathrm{SUU}}$ and $\mathrm{tRNA}^{\mathrm{fMet}}{ }_{\mathrm{CAU}}$ from E. coli were purchased from Chemical Block (Russia). The mRNA constructs whose sequences are specified below were from Thermo Scientific (USA) and deprotected following the supplier procedure. All mRNA constructs contained identical sequence $5^{\prime}$-GGCAAGGAGGUAAAA- $3^{\prime}$ at the $5^{\prime}$-end, which was followed by $5^{\prime}$-AUGAAAA - $^{\prime} 3^{\prime}$ (mRNA-1), $5^{\prime}$-AUGAAGA A $_{9} 3^{\prime}$ (mRNA-2), 5' -AUGUAAA $9-3^{\prime}$ (mRNA-3) or $5^{\prime}$-AUGAUAA ${ }_{9}-3^{\prime}$ (mRNA-4). Aminoglycoside antibiotic paromomycin was purchased from Sigma-Aldrich.}

Purification of the ribosomes. Purification of the 70S ribosomes from strain HB8 of T. thermophilus was performed according to the protocol described in ref. 30 .

Complex formation. All ribosomal complexes were formed in $10 \mathrm{mM}$ Tris-acetate $\mathrm{pH} 7.0,40 \mathrm{mM} \mathrm{KCl}, 7.5 \mathrm{mM} \mathrm{Mg}\left(\mathrm{CH}_{3} \mathrm{COO}\right)_{2}, 0.5 \mathrm{mM}$ dithiothreitol at $37^{\circ} \mathrm{C}$.
For the cognate complexes (Fig. 1c, complexes 1 and 2), the 70 S ribosomes $(3 \mu \mathrm{M})$ were pre-incubated with fivefold excess of mRNA-1 or mRNA-2 and threefold excess of $\mathrm{tRNA}^{\mathrm{fMet}}{ }_{\mathrm{CAU}}$ for $15 \mathrm{~min}$ to fill the P-site. Then, $\mathrm{tRNA}^{\mathrm{Lys}}{ }_{\text {SUU }}$ was added at fivefold excess and incubation was continued for $30 \mathrm{~min}$. Near-cognate complexes (Fig. 1c, complexes 3 and 4) were prepared in a similar manner with the use of mRNA-3 and mRNA-4 constructs. Complexes with paromomycin were obtained by including the antibiotic $(60 \mu \mathrm{M})$ into the incubation mixture containing 70S/tRNA ${ }^{\text {fMet }} / \mathrm{mRNA}-3 / \mathrm{tRNA}^{\mathrm{Lys}}{ }_{\text {SUU }}$ or $70 \mathrm{~S} / \mathrm{tRNA}^{\mathrm{fMet}} / \mathrm{mRNA} 4 / \mathrm{tRNA}^{\text {Lys }}$ suU.

Crystallization and crystal treatment. Crystals were grown at $24^{\circ} \mathrm{C}$ via vapour diffusion in sitting-drop plates (CrysChem, Hampton Research). The ribosomal complex $(2 \mu \mathrm{l})$ containing $2.8 \mathrm{mM}$ Deoxy Big Chaps (CalBioChem) was mixed with the equal volume of the crystallization solution (3.9-4.2\% (w/v) PEG 20k, 3.9-4.2\% (w/v) PEG550mme, $100 \mathrm{mM}$ Tris-acetate pH 7.0, $100 \mathrm{mM}$ KSCN). The crystals grew for 2-3 weeks and were then dehydrated by exchanging the reservoir for $60 \%$ (v/v) 2-methyl-2,4-pentanediol. Before freezing in the nitrogen stream, crystals were then cryoprotected by the addition of $30 \%(\mathrm{v} / \mathrm{v})$ 2-methyl-2,4-pentanediol and $14 \mathrm{mM} \mathrm{Mg}\left(\mathrm{CH}_{3} \mathrm{COO}\right)_{2}$.

Structure determination. Data for all complexes were collected at the PXI beamline of Swiss Light Source, Switzerland, at $100 \mathrm{~K}$. A very low-dose mode was used and high redundancy data were collected ${ }^{52}$. The data were processed and scaled using XDS ${ }^{53}$. All crystals belong to space group $\mathrm{P} 2{ }_{1} 2_{1} 2_{1}$ and contain two ribosomes per asymmetric unit. One of the previously published structures ${ }^{29}$, with tRNA, mRNA and metal ions removed, was used for refinement with Phenix ${ }^{54}$. The initial model was placed within each data set by rigid body refinement with each biopolymer chain as a rigid body. This was followed by initial coordinate refinement. The resulting electron density maps were inspected in $\operatorname{Coot}^{55}$, and the tRNA and mRNA ligands were built in. During several cycles of manual rebuilding followed by coordinate and isotropic B-factor refinement, magnesium ions were added and the final refinement round took place. The data collection and refinement statistics are presented in Table 1.

\section{References}

1. Machnicka, M. A. et al. MODOMICS: a database of RNA modification pathways--2013 update. Nucleic Acids Res. 41, D262-D267 (2013).

2. El Yacoubi, B., Bailly, M. \& de Crecy-Lagard, V. Biosynthesis and function of posttranscriptional modifications of transfer RNAs. Annu. Rev. Genet. 46, 69-95 (2012)

3. Nishimura, S. \& Watanabe, K. The discovery of modified nucleosides from the early days to the present: a personal perspective. J. Biosci. 31, 465-475 (2006).

4. Agris, P. F., Vendeix, F. A. \& Graham, W. D. tRNA's wobble decoding of the genome: 40 years of modification. J. Mol. Biol. 366, 1-13 (2007).

5. Cantara, W. A. et al. The RNA modification database, RNAMDB: 2011 update. Nucleic Acids Res. 39, D195-D201 (2011).

6. Durant, P. C., Bajji, A. C., Sundaram, M., Kumar, R. K. \& Davis, D. R. Structural effects of hypermodified nucleosides in the Escherichia coli and human tRNALys anticodon loop: the effect of nucleosides s $2 \mathrm{U}, \mathrm{mcm} 5 \mathrm{U}$, mcm5s2U, mnm5s2U, t6A, and ms2t6A. Biochemistry 44, 8078-8089 (2005).

7. Stuart, J. W., Koshlap, K. M., Guenther, R. \& Agris, P. F. Naturally-occurring modification restricts the anticodon domain conformational space of tRNA(Phe). J. Mol. Biol. 334, 901-918 (2003).

8. Crick, F. H. Codon-anticodon pairing: the wobble hypothesis. J. Mol. Biol. 19, 548-555 (1966).

9. Yarian, C. et al. Accurate translation of the genetic code depends on tRNA modified nucleosides. J. Biol. Chem. 277, 16391-16395 (2002).

10. Bjork, G. R. et al. Transfer RNA modification. Annu. Rev. Biochem. 56, 263-287 (1987).

11. Agris, P. F. Wobble position modified nucleosides evolved to select transfer RNA codon recognition: a modified-wobble hypothesis. Biochimie 73, 1345-1349 (1991).

12. Beuning, P. J. \& Musier-Forsyth, K. Transfer RNA recognition by aminoacyl-tRNA synthetases. Biopolymers 52, 1-28 (1999).

13. Grosjean, H. \& Bjork, G. R. Enzymatic conversion of cytidine to lysidine in anticodon of bacterial isoleucyl-tRNA--an alternative way of RNA editing. Trends Biochem. Sci. 29, 165-168 (2004).

14. Bjork, G. R. et al. Transfer RNA modification: influence on translational frameshifting and metabolism. FEBS Lett. 452, 47-51 (1999).

15. Urbonavicius, J., Qian, Q., Durand, J. M., Hagervall, T. G. \& Bjork, G. R. Improvement of reading frame maintenance is a common function for several tRNA modifications. EMBO J. 20, 4863-4873 (2001)

16. Thiaville, P. C. \& de Crecy-Lagard, V. The emerging role of complex modifications of tRNA in signaling pathways. Microb. Cell 2, 1-4 (2015).

17. Kellogg, D. A., Doctor, B. P., Loebel, J. E. \& Nirenberg, M. W. RNA codons and protein synthesis. IX. Synonym codon recognition by multiple species of valine-, alanine-, and methionine-sRNA. Proc. Natl Acad. Sci. USA 55, 912-919 (1966).

18. Crick, F. H. The origin of the genetic code. J. Mol. Biol. 38, 367-379 (1968). 
19. Raba, M. et al. Nucleotide sequence of three isoaccepting lysine tRNAs from rabbit liver and SV40-transformed mouse fibroblasts. Eur. J. Biochem. 97, 305-318 (1979).

20. Sprinzl, M. \& Vassilenko, K. S. Compilation of tRNA sequences and sequences of tRNA genes. Nucleic Acids Res. 33, D139-D140 (2005).

21. Armengod, M. E. et al. Enzymology of tRNA modification in the bacterial MnmEG pathway. Biochimie 94, 1510-1520 (2012).

22. Kumar, R. K. \& Davis, D. R. Synthesis and studies on the effect of 2-thiouridine and 4-thiouridine on sugar conformation and RNA duplex stability. Nucleic Acids Res. 25, 1272-1280 (1997).

23. Ogle, J. M. et al. Recognition of cognate transfer RNA by the $30 \mathrm{~S}$ ribosomal subunit. Science 292, 897-902 (2001).

24. Murphy, F. V. T. \& Ramakrishnan, V. Structure of a purine-purine wobble base pair in the decoding center of the ribosome. Nat. Struct. Mol. Biol. 11, 1251-1252 (2004).

25. Murphy, F. V. t., Ramakrishnan, V., Malkiewicz, A. \& Agris, P. F. The role of modifications in codon discrimination by tRNA(Lys)UUU. Nat. Struct. Mol. Biol. 11, 1186-1191 (2004).

26. Vendeix, F. A. et al. Human tRNA(Lys3)(UUU) is pre-structured by natural modifications for cognate and wobble codon binding through keto-enol tautomerism. J. Mol. Biol. 416, 467-485 (2012).

27. Weixlbaumer, A. et al. Mechanism for expanding the decoding capacity of transfer RNAs by modification of uridines. Nat. Struct. Mol. Biol. 14, 498-502 (2007).

28. Jenner, L. B., Demeshkina, N., Yusupova, G. \& Yusupov, M. Structural aspects of messenger RNA reading frame maintenance by the ribosome. Nat. Struct. Mol. Biol. 17, 555-560 (2010).

29. Demeshkina, N., Jenner, L., Westhof, E., Yusupov, M. \& Yusupova, G. A new understanding of the decoding principle on the ribosome. Nature 484, 256-259 (2012).

30. Rozov, A., Demeshkina, N., Westhof, E., Yusupov, M. \& Yusupova, G. Structural insights into the translational infidelity mechanism. Nat. Commun. 6, 7251 (2015).

31. Selmer, M. et al. Structure of the $70 \mathrm{~S}$ ribosome complexed with mRNA and tRNA. Science 313, 1935-1942 (2006).

32. Fernandez, I. S. et al. Unusual base pairing during the decoding of a stop codon by the ribosome. Nature 500, 107-110 (2013).

33. Schmeing, T. M., Voorhees, R. M., Kelley, A. C. \& Ramakrishnan, V. How mutations in tRNA distant from the anticodon affect the fidelity of decoding. Nat. Struct. Mol. Biol. 18, 432-436 (2011).

34. Holley, R. W. et al. Structure of a ribonucleic acid. Science 147, 1462-1465 (1965).

35. Ogle, J. M., Murphy, F. V., Tarry, M. J. \& Ramakrishnan, V. Selection of tRNA by the ribosome requires a transition from an open to a closed form. Cell 111, $721-732$ (2002)

36. Yokoyama, S. et al. Molecular mechanism of codon recognition by tRNA species with modified uridine in the first position of the anticodon. Proc. Natl Acad. Sci. USA 82, 4905-4909 (1985).

37. Mazumdar, S. K. \& Saenger, W. Molecular structure of poly-2-thiouridylic acid, a double helix with non-equivalent polynucleotide chains. J. Mol. Biol. 85, 213-219 (1974)

38. Demeshkina, N., Jenner, L., Yusupova, G. \& Yusupov, M. Interactions of the ribosome with mRNA and tRNA. Curr. Opin. Struct. Biol. 20, 325-332 (2010).

39. Nakamura, Y. Codon Usage Database http://www.kazusa.or.jp/codon/ (2007).

40. Frédéric Dardel, F. K. Bioinformatics: Genomics and Post-Genomics (John Wiley \& Sons, Ltd., 2006).

41. Takai, K. \& Yokoyama, S. Roles of 5-substituents of tRNA wobble uridines in the recognition of purine-ending codons. Nucleic Acids Res. 31, 6383-6391 (2003).

42. Egli, M. \& Sarkhel, S. Lone pair-aromatic interactions: to stabilize or not to stabilize. Acc. Chem. Res. 40, 197-205 (2007).

43. Wohlgemuth, I., Pohl, C., Mittelstaet, J., Konevega, A. L. \& Rodnina, M. V. Evolutionary optimization of speed and accuracy of decoding on the ribosome. Philos. Trans. R. Soc. Lond. B Biol. Sci. 366, 2979-2986 (2011).

44. Kramer, E. B. \& Farabaugh, P. J. The frequency of translational misreading errors in E. coli is largely determined by tRNA competition. RNA 13, 87-96 (2007).

45. Johansson, M., Zhang, J. \& Ehrenberg, M. Genetic code translation displays a linear trade-off between efficiency and accuracy of tRNA selection. Proc. Natl Acad. Sci. USA 109, 131-136 (2012).
46. Manickam, N., Nag, N., Abbasi, A., Patel, K. \& Farabaugh, P. J. Studies of translational misreading in vivo show that the ribosome very efficiently discriminates against most potential errors. RNA 20, 9-15 (2014).

47. Moazed, D. \& Noller, H. F. Binding of tRNA to the ribosomal A and P sites protects two distinct sets of nucleotides in 16S rRNA. J. Mol. Biol. 211, 135-145 (1990).

48. Demeshkina, N., Jenner, L., Westhof, E., Yusupov, M. \& Yusupova, G. New structural insights into the decoding mechanism: translation infidelity via a G.U pair with Watson-Crick geometry. FEBS Lett. 587, 1848-1857 (2013).

49. Westhof, E., Yusupov, M. \& Yusupova, G. Recognition of Watson-Crick base pairs: constraints and limits due to geometric selection and tautomerism. F1000Prime Rep. 6, 19 (2014).

50. Grosjean, H., de Crecy-Lagard, V. \& Marck, C. Deciphering synonymous codons in the three domains of life: co-evolution with specific tRNA modification enzymes. FEBS Lett. 584, 252-264 (2010).

51. Jenner, L., Demeshkina, N., Yusupova, G. \& Yusupov, M. Structural rearrangements of the ribosome at the tRNA proofreading step. Nat. Struct. Mol. Biol. 17, 1072-1078 (2010).

52. Mueller, M., Wang, M. \& Schulze-Briese, C. Optimal fine phi-slicing for single-photon-counting pixel detectors. Acta Crystallogr. D Biol. Crystallogr. 68, 42-56 (2012).

53. Kabsch, W. Xds. Acta Crystallogr. D Biol. Crystallogr. 66, 125-132 (2010)

54. Adams, P. D. et al. PHENIX: a comprehensive Python-based system for macromolecular structure solution. Acta Crystallogr. D Biol. Crystallogr. 66, 213-221 (2010).

55. Emsley, P., Lohkamp, B., Scott, W. G. \& Cowtan, K. Features and development of Coot. Acta Crystallogr. D Biol. Crystallogr. 66, 486-501 (2010).

56. Karplus, P. A. \& Diederichs, K. Linking crystallographic model and data quality. Science 336, 1030-1033 (2012).

\section{Acknowledgements}

We thank the staff of the PXI beamline at the Swiss Light Source (Switzerland) for the advices in the synchrotron data collection. We are also thankful to Henry Grosjean for critical reading of the manuscript and constructive discussions. This work was supported by the French National Research Agency ANR-11-BSV8-006 01 (to G.Y.), the European Research Council advanced grant 294312 (to M.Y.), the Foundation for Medical Research in France grant FDT20140930867 (to I.K.) and the Russian Government Program of Competitive Growth of Kazan Federal University (to I.K., M.Y. and G.Y.). This work was also supported by the LABEX: ANR-10-LABX-0036_NETRNA and benefits from a funding from the state managed by the French National Research Agency as part of the Investments for the future program (to E.W.)

\section{Author contributions}

A.R. and N.D. conducted the experiments. I.K. purified ribosomes and assisted in X-ray data collection. A.R., N.D., E.W. and G.Y. interpreted the structures. A.R., N.D., I. K., E.W., M. Y. and G.Y. contributed to the final version of the paper.

\section{Additional information}

Accession codes: The atomic coordinates and structure factors for the reported crystal structures have been deposited in the Protein Data Bank under accession codes: 5E7K, 5E81, 5EL4, 5EL5, 5EL6 and 5EL7.

Supplementary Information accompanies this paper at http://www.nature.com/ naturecommunications

Competing financial interests: The authors declare no competing financial interests.

Reprints and permission information is available online at http://npg.nature.com/ reprintsandpermissions/

How to cite this article: Rozov, A. et al. Novel base-pairing interactions at the tRNA wobble position crucial for accurate reading of the genetic code. Nat. Commun. 7:10457 doi: 10.1038/ncomms10457 (2016).

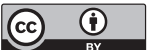

This work is licensed under a Creative Commons Attribution 4.0 International License. The images or other third party material in this article are included in the article's Creative Commons license, unless indicated otherwise in the credit line; if the material is not included under the Creative Commons license, users will need to obtain permission from the license holder to reproduce the material. To view a copy of this license, visit http://creativecommons.org/licenses/by/4.0/ 